Association for Information Systems AIS Electronic Library (AISeL)

Wirtschaftsinformatik Proceedings 2003

Wirtschaftsinformatik

September 2003

\title{
A Model for Inter-Organizational Business Process Integration
}

Boris Otto

Fraunhofer Institute for Industrial Engineering, boris.ott@iao.fraunhofer.de

Jürgen Wäsch

e-pro solutions $\mathrm{GmbH}$

Follow this and additional works at: http://aisel.aisnet.org/wi2003

\section{Recommended Citation}

Otto, Boris and Wäsch, Jürgen, "A Model for Inter-Organizational Business Process Integration" (2003). Wirtschaftsinformatik Proceedings 2003. 23.

http://aisel.aisnet.org/wi2003/23

This material is brought to you by the Wirtschaftsinformatik at AIS Electronic Library (AISeL). It has been accepted for inclusion in Wirtschaftsinformatik Proceedings 2003 by an authorized administrator of AIS Electronic Library (AISeL). For more information, please contact elibrary@aisnet.org. 
In: Uhr, Wolfgang, Esswein, Werner \& Schoop, Eric (Hg.) 2003. Wirtschaftsinformatik 2003: Medien - Märkte - Mobilität, 2 Bde. Heidelberg: Physica-Verlag

ISBN: 3-7908-0111-9 (Band 1)

ISBN: 3-7908-0116-X (Band 2)

(C) Physica-Verlag Heidelberg 2003 


\title{
A Model for Inter-Organizational Business Process Integration
}

\section{Boris Otto}

Fraunhofer Institute for Industrial Engineering

\section{Jürgen Wäsch}

e-pro solutions $\mathrm{GmbH}$

\begin{abstract}
The present paper proposes an integrated model for interorganizational business process integration. The model does not just tackle the execution of processes, but takes into account also the integration process itself, which has turned out to be a key cost driver in many integration projects. After the identification of the market needs, the state of the art is illustrated in terms of technologies and methodologies that facilitate business process integration. Based on this, the model is decomposed into three different layers: 'integration phases', 'level of implementation' and 'actor roles'. The paper concludes with an integrated view on the model and the identification of current shortcomings and future fields of research.
\end{abstract}

Keywords: business process integration, Web Services, business process frameworks, process components

\section{Introduction}

In recent years it turned out that the main achievement of electronic business solutions is the reduction of costs that companies spend for the design and execution of business processes between their business partners and their internal departments. The need for cost reduction gets even more important as many companies see themselves confronted with the effects of globalized markets. Therefore, the reduction of process costs is not just an opportunity that may be taken, but rather a conditio sine qua non.

In all industrial sectors, collaborative arrangements have developed from monolithic, tightly-coupled supply chains to dynamic, highly integrated value-added networks that consist of competence nodes formed by different organizations or parts of organizations. The trend still prevails to outsource non-core functionality to external partners and to leverage the positive effects of economies of scale that 
can be achieved by integrated networks [cp. VDA02, p. 58]. To cope with current challenges, these networks must have a highly dynamic organizational structure [Bull99, p. 11].

Basically, for companies to stay competitive it is mandatory to achieve two concurrent goals, namely the reduction of costs for end-to-end business processes on the one hand and the provisioning of a very sophisticated process model that supports the requirements of dynamic networks on the other hand. Considering this, efficient technologies and methodologies for the integration of business processes form the key success factor to achieve both goals.

In the following, a comprehensive model for inter-organizational business process integration will be developed that is based on existing approaches, such as Web Services and business process frameworks (mainly ebXML). Also, the model will take into account the semantic dimension of business processes.

\section{Market and User Needs}

Several market and user needs can be identified that force companies to adapt their business processes accordingly.

Nowadays, it is extremely important that two - or even more - companies can do business 'on the fly', i.e. that the integration of business processes of different companies can be realized in an automated way, without extensive preparation and with easy disconnection after the business relationship - which may have been set up as a temporary arrangement from the outset - has ended. Moreover, it must be taken into account that dynamic networks do not only consist of major players with flexible process models and sophisticated information technology (IT) infrastructures, but also of small and medium sized enterprises (SMEs). Thus a model for efficient business process integration must also take account of the needs of less developed companies. If the model does not live up to specific SME requirements, improvements may be achieved on the side of large companies, but certainly not along the entire supply chain. Therefore, appropriate approaches must be affordable to SMEs, too [Stef02, p. 145]. Also, dynamic networks require dynamic processes in terms of process control, i.e. a process triggered in one company might consist of process components that are beyond the control of that company, but are assembled at runtime. This concept is completely different from the deterministic process chains that usually can be found between companies today $[\mathrm{Kli}+99$, p. 5]. Apart from that, an integrated model must not be limited to the processes within an organization. It is rather the main characteristic of modern business process management to cover process chains from the initiation in one organization until the finalization in another organization. Therefore, the integration of modern system architectures - usually deployed for the execution of inter- 
organizational processes - with legacy systems - such as Enterprise Resource Planning (ERP) or transaction systems - must be supported. Furthermore, it is essential to close the gap between the modeling view of business process management and the implementation view. This leads to the combination of a business process level and a system level within the same model.

\section{State of the Art}

Derived from the overall market needs, the following chapter gives a brief overview of the technological state of the art in the field of business process integration. Areas related to this are mainly business process frameworks, process modeling methodologies, the so-called Web Services stack and semantic technologies.

\subsection{Business Process Frameworks}

Business process frameworks aim at providing standardized inter-organizational process models in order to reduce the complexity and the costs of process modeling and (re-)engineering. They often enhance pure business document specifications [Fran01, p. 287]. The following parameters can be applied to analyze and evaluate different initiatives:

- Target sector: Some frameworks were developed especially for the use in a particular industrial sector, whereas others are of a broader scope. The first have a vertical dimension, the latter a horizontal one [EsZu02, p. 253].

- Consortium composition: Different types of consortia can be identified. Some consist of industry representatives, some are driven by software vendors, and others are developed under the umbrella of an independent standardization organization, e.g. CEN, OASIS or UN/CEFACT.

- Functional completeness: Some initiatives just offer limited functionality - in terms of business processes - for specific application scenarios, whereas others cover entire business areas.

- Registry and repository: Another evaluation parameter derives from the provisioning of mechanisms for registries to store company and service information as well as for repositories to store process schemas.

- Relation to document specifications: Since the objective of a lot of initiatives is the provisioning of a framework in which business documents are exchanged, it is an important criterion as to which business documents specifications are compatible. 
- Implementation model: It is important to distinguish between initiatives that come up with an implementation guideline or even software support from those that do not [cp. EsZu02, p. 253]. This differentiation helps to close the gap between modeling and implementing business processes.

- Acceptance: The user acceptance regarding a standardized process framework must be considered when evaluating different initiatives in order to minimize investment risks and to maximize future applicability.

In the following, relevant initiatives are introduced in brief and evaluated against the above criteria.

- ebXML: ebXML is an initiative that aims at the development of an XMLbased infrastructure to facilitate efficient electronic business. The scope covers both technical aspects of the infrastructure and business process related parts. ebXML was launched in 1999 and is now hosted by OASIS and UN/CEFACT. Those working groups that deal with the technical infrastructure (Messaging Services, Registries and Repositories, Collaborative Protocol Profile and Implementation, Interoperability and Conformance) are located within OASIS, whereas those that deal with the business process layer (Core Components and Business Process Models) are located within UN/CEFACT. The framework provides specifications and scenarios for the use of ebXML in UDDI environments [Somm02].

- RosettaNet: In contrast to ebXML, RosettaNet is a vertical approach to create a standard for electronic business for the information technology, electronic components and semiconductor manufacturing industries. RosettaNet is a non-profit organization that covers the needs of the entire supply chain. RosettaNet provides so-called Partner Interface Processes (PIPs), that specify the business processes between different partners, and a technical infrastructure for their execution. The latter is combined within the RosettaNet Implementation Framework (RNIF) [Rose02]. Since August 2002, RosettaNet is an official subsidiary of the Uniform Code Council, Inc. (UCC).

- WSCI: The Web Services Choreography Interface describes the flow of messages exchanged between different Web Services. The WSCI specification was developed by a consortium of private companies, among them Sun Microsystems, SAP, and BEA Systems, but also forms the foundation for the W3C Web Services Choreography Working Group. The focus of WSCI clearly lies on the inter-organizational part of end-to-end business processes [Ark+02].

- BizTalk: BizTalk is Microsoft's approach to provide a solution to facilitate inter-organizational electronic business interoperability. Microsoft argues that there is no common standard that covers all business needs so that a transformation between different approaches still is - and probably will be in the future - necessary in order to establish efficient business processes between different partners. The BizTalk Framework is currently available as version 2.0 
that comprises the notions of BizTalk Document and BizTalk Message [Micr02]. The framework can be supported by $3^{\text {rd }}$-party products also, but is very much synchronized to the BizTalk Server.

- WSFL: The Web Services Flow Language is IBM's formal approach to describe the composition of Web Services. The result of such compositions can either be a complete business process that consists of single Web Services or a so-called interaction pattern to describe the interaction of different business partners at runtime [Leym01]. WSFL and Microsoft's XLANG have now converged to BPEL4WS [WeCu02].

- BPEL4WS: The Business Process Execution Language for Web Services is a joint initiative of BEA Systems, IBM and Microsoft to specify business process behavior based on the concept of Web Services. BPEL4WS will supersede WSFL and XLANG in the medium term. A BPEL4WS process consists of several elements, i.e. 'partners', 'containers' for the payloads, 'faultHandlers' for exceptions from the regular process, and 'activities' that form the business process [And+03].

The evaluation of these initiatives against the above mentioned criteria is given in Table $1^{1}$.

\begin{tabular}{|c|c|c|c|c|c|c|}
\hline Criterion & $\begin{array}{l}\sum_{0}^{1} \\
\text { O }\end{array}$ & 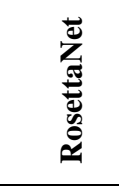 & 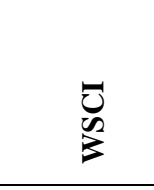 & 兴 & $\begin{array}{l}\overrightarrow{1} \\
\frac{1}{3} \\
3\end{array}$ & $\sum_{i=1}^{\infty}$ \\
\hline Target Sector & no specific & $\begin{array}{l}\text { IT, EC, } \\
\text { SM }\end{array}$ & no specific & $\begin{array}{l}\text { no spe- } \\
\text { cific }\end{array}$ & $\begin{array}{l}\text { no spe- } \\
\text { cific }\end{array}$ & $\begin{array}{l}\text { no spe- } \\
\text { cific }\end{array}$ \\
\hline $\begin{array}{l}\text { Consortium } \\
\text { Composition }\end{array}$ & $\begin{array}{l}\text { non-profit, } \\
\text { independent }\end{array}$ & $\begin{array}{c}\text { non- } \\
\text { profit, } \\
\text { sectoral }\end{array}$ & $\begin{array}{l}\text { non-profit, } \\
\text { vendor } \\
\text { dominated }\end{array}$ & $\begin{array}{c}\text { Micro- } \\
\text { soft }\end{array}$ & IBM & $\begin{array}{c}\text { BEA, } \\
\text { IBM, } \\
\text { Micro- } \\
\text { soft, SAP }\end{array}$ \\
\hline $\begin{array}{l}\text { Functional Com- } \\
\text { pleteness }\end{array}$ & + & + & \pm & + & - & \pm \\
\hline $\begin{array}{l}\text { Registry and Re- } \\
\text { pository }\end{array}$ & + & - & - & - & - & - \\
\hline $\begin{array}{l}\text { Document Spec's } \\
\text { Relation }\end{array}$ & $\begin{array}{c} \pm \\
(\mathrm{PIP}, \mathrm{xCBL})\end{array}$ & $\begin{array}{c}+ \\
(\mathrm{PIPs}) \\
\end{array}$ & - & - & - & - \\
\hline Implementation & + & + & - & + & \pm & \pm \\
\hline
\end{tabular}

1 For lack of space, the parameter values and the evaluation respectively are presented in a condensed format. This procedure is also applied for the subsequent state-of-theart chapters. 


\begin{tabular}{|c|c|c|c|c|c|c|}
\hline Criterion & $\begin{array}{l}\sum_{0} \\
\text { O } \\
0\end{array}$ & 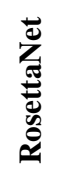 & $\begin{array}{l}\tilde{Z} \\
\mathscr{K}\end{array}$ & 音 & $\begin{array}{l}\overrightarrow{1} \\
\overrightarrow{3} \\
\overrightarrow{3}\end{array}$ & 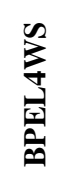 \\
\hline \multicolumn{7}{|l|}{ Model } \\
\hline Acceptance & \pm & + & - & \pm & - & - \\
\hline
\end{tabular}

IT: information technology; EC: electronic components; SM: semiconductor manufacturing; PIP: Partner Interface Process; + : high parameter value; \pm : medium parameter value ; - : low parameter value.

Table 1: Evaluation of business process frameworks

Table 1 shows that no initiative fulfils all requirements for business process frameworks. Most approaches fall short with regard to the provisioning of registries and repositories, whereas the most complete one - ebXML - is still under development.

\subsection{Business Process Modeling Methodologies}

Methodologies for the modeling of business processes form the link between a business driven view on a business process and its formal representation that can be implemented in an application system. They are a critical element of efficient business process management. However, there is still a variety of different approaches; no standard methodology for business process modeling can be identified at present [Rose00]. Their applicability for different usage scenarios can be evaluated according to the following set of parameters:

- Main application area: Depending on the focus of the methodology, it can be distinguished between data oriented, process oriented, system oriented and organizational approaches. Also, approaches that originally were developed in the field of software engineering turned out to be helpful in terms of process modeling [BuFä97].

- Degree of formalization: Modeling methodologies can be divided in nonformal ones that can be easily used also by people with no or few technical skills and formal ones that can be used for the specification of information systems and process models that are machine readable [Kur+97].

- Structural flexibility: This evaluation parameter represents the methodology's ability to support also complex processes (sequences, parallel processes, alternative processes and iterative processes) [ScTü99, p. 521].

- Tool support: The practical applicability of a methodology highly depends on the existence of adequate support by software tools [cp. BuSc01]. 
- User acceptance: Especially in collaborative environments, user acceptance is essential for both the reduction of investment risks and the efficiency of information exchange between partners.

- Standards compliance: Since the modeling result should be a machine readable process representation, it is important that the modeling methodology is compliant to existing standards in the field of business process frameworks.

The following modeling methodologies are evaluated against their ability to support efficient business process integration [cp. open02a]:

- ARIS: Architecture of Integrated Information Systems is a method for the design and description of information systems that support business processes. It is based on a decomposition of the entire subject into a process, an organization and a data view. ARIS also provides a notation for modeling business processes, i.e. Event-Driven Process Chains (EPC) [Sche98]. Since the method was developed with sound consideration of the enterprise model that forms the foundation of SAP's R/3 System, ARIS is widely used in the user community, especially in Germany.

- UML: The Unified Modeling Language is an object-oriented approach for the development of application systems. Thanks to its wide-spread use, however, UML is also often applied for business process modeling. UML emerged from the three approaches of Booch, Jacobson and Rumbaugh and was standardized in 1997 by the Object Management Group (OMG). UML recognizes a number of different diagrams, i.e. 'class diagrams', 'use cases', 'interaction diagrams', 'collaboration diagrams', 'state charts', 'activity diagrams', and 'implementation diagrams' [OMG01].

- UMM: The UN/CEFACT Modeling Methodology is the formal description technique for describing any Open-EDI scenario as defined in ISO/IEC IS 14662. The primary focus of UMM is the Business Operations View (BOV) and not the Functional Service View (FSV). UMM provides a procedure for specifying - in a technology neutral, implementation independent manner business processes involving information exchange. UMM recognizes four general phases of business re-engineering and software development projects, i.e. 'inception', 'elaboration', 'construction', and 'transition'. UMM focuses on the first two phases and provides four workflows ('business modeling', 'requirements', 'analysis', and 'design') to facilitate the formal modeling of business processes [UN/C01].

- Petri Nets: Petri Nets are a formal mechanism for defining concurrent processes. They were mainly introduced to model technical processes but are also easy to use for the definition of business processes. One of the biggest advantages is the formalism of the modeling method which can be translated into Event Driven Process Chains and UML diagrams. Petri Nets are characterized by a strong differentiation between passive and active system components, rep- 
resented through passive and active network elements, called transitions. The passive transitions represent local states of the system, which can be valid during the system's runtime. Transitions represent actions and events, which have specific states as a precondition for occurrence. From the transitions point of view, the states are conditions. Transitions and states are linked together by flows [cp. Petr62]

The evaluation of the modeling methodologies against the evaluation criteria is given in Table 2:

\begin{tabular}{|c|c|c|c|c|}
\hline Criterion & $\frac{\mathscr{2}}{2}$ & $\sum$ & $\sum^{\sum}$ & 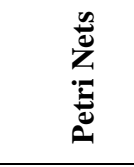 \\
\hline Main Application Area & processes & $\begin{array}{c}\text { data, organiza- } \\
\text { tions, proc- } \\
\text { esses }\end{array}$ & $\begin{array}{c}\text { organizations, } \\
\text { processes }\end{array}$ & processes \\
\hline Degree of Formalization & \pm & + & \pm & + \\
\hline Structural Flexibility & + & \pm & \pm & + \\
\hline Tool Support & + & + & - & \pm \\
\hline User Acceptance & + & + & - & \pm \\
\hline $\begin{array}{l}\text { Compliance to Business } \\
\text { Process Frameworks }\end{array}$ & - & \pm & $\begin{array}{c}+ \\
(\mathrm{ebXML})\end{array}$ & - \\
\hline
\end{tabular}

+ : high parameter value; \pm : medium parameter value $;-:$ low parameter value.

Table 2: Evaluation of business process modeling methodologies

The table shows that only UMM is clearly synchronized with a standardization initiative for business process frameworks. However, its acceptance in the user community is still low. This leads to the assumption that business process frameworks still have shortcomings in terms of support by a widely used, formal modeling methodology, although the latter is essential for efficient inter-organizational business process management.

\subsection{The Web Services Stack}

Web Services are a promising effort to overcome current shortcomings of collaborative system infrastructures. The Web Services Activity of the W3C defines Web Services as programmatic interfaces for application-to-application communication [W3C03]. Web Services are programmable, self-describing, encapsulated and loosely coupled [Bett01, p. 302]. The focus of Web Services lies therefore on the inter-organizational coupling of business functionality using standard web proto- 
cols and formats. Taking this into consideration, Web Services can also be defined as parts of specific business functions that are visible 'from outside' by being exposed to the Web via well-defined interfaces and that are invoked via standard Web protocols [Fen+01, p. 11]. The most prominent and relevant ones are referred to as the 'Web Services Stack', which consists of the following standards:

- SOAP: The Simple Object Access Protocol is a protocol that describes how structured information between network nodes can be exchanged as XML messages within distributed environments, hence it enables remote procedure calls over the Web. A SOAP message basically consists of three parts, namely an 'envelope', a 'header', and a 'body' that contains the actual business information [cp. Gud+02].

- WSDL: The Web Services Description Language provides a model and an XML based language to describe Web Services [Chi+03]. All Web Services form a node within an entire network of services. A WSDL compliant description contains all pieces of information that a client needs to access the business functionality that is represented by the Web Service.

- UDDI: Based on the technological foundations of SOAP and WSDL that enable the basic communication between different applications over the Web, UDDI (Universal Description, Discovery and Integration) specifies a registry that provides information as to which Web Services are available and by whom they are offered. For the representation of information, UDDI recognizes two basic elements, i.e. 'businessEntity' and 'businessService'.

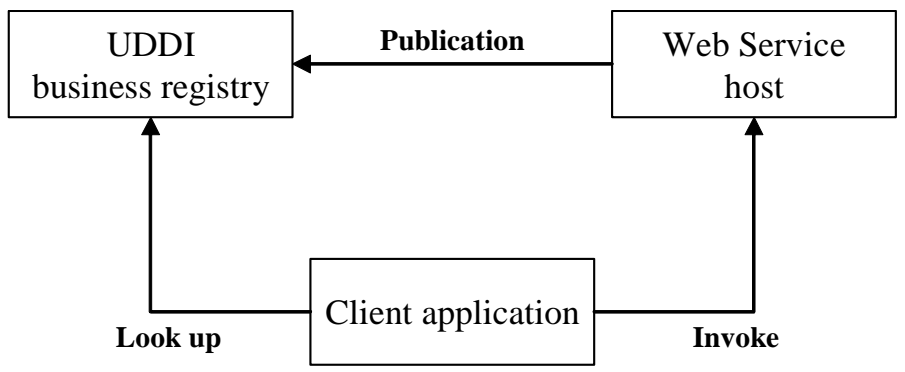

Figure 1: The Web Services concept

The basic concept of Web Services and the relationship between the three elements of the Web Services stack is described in Figure 1 [cp. WoWe02, p. 108]. The Web Services idea provides a promising technical foundation for efficient business process integration because it separates the business functionality from the underlying system infrastructure. However, shortcomings still exist. Up to now, it is not clear under which business models registries can be operated, how Web Services quality can be measured, and so forth. 


\subsection{Semantic Technologies}

Closely related to Web Services technologies is the concept of the Semantic Web. Due to the fact that Web Services are supposed to foster automated machine-tomachine communication over the Web, the Semantic Web addresses the problem of different interpretation of the content that is available on the Internet. The Semantic Web aims at describing the semantics of information resources on the Internet and their relationships to each other. In other words, the Semantic Web is an approach for specifying an additional layer of information description, namely the semantic layer, that is based on the syntactic layer [ScFi02, p. 18].

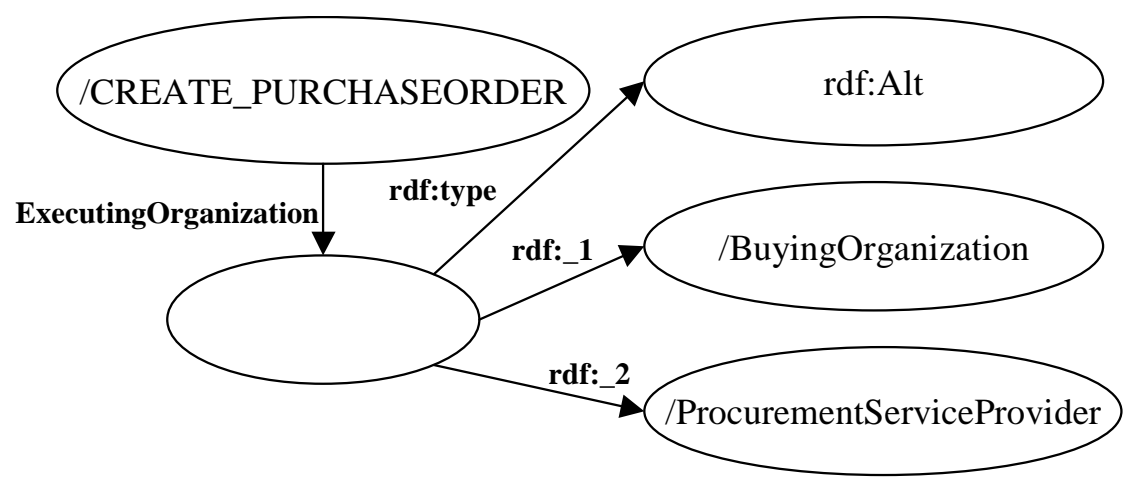

Figure 2: Exemplary RDF model

Among the basic technologies that enable the Semantic Web are:

- RDF: The Resource Description Framework is a model that uses XML as a syntax to describe meta-data, hence enabling the linkage between the syntactical and the semantic description of information resources [LaSw99]. RDF consists of three basic types of objects, i.e. 'resources', 'properties', and 'statements'. Moreover, so-called RDF containers ('bags', 'sequences' and 'alternatives') are introduced to refer to a collection of resources rather than to a single resource. The RDF model is enhanced by the RDF Schema that provides mechanisms to describe RDF properties and the relationships between them [BrGu03]. An RDF example is given in the figure above in which the sentence 'The process component CREATE_PURCHASEORDER can be executed by a buying organization or a procurement service provider' is represented as an RDF model.

- Ontologies: An ontology can be defined as a shared, abstract model of some phenomenon in the world that is explicitly described and formalized in a way that it is also understandable by machines. Ontologies can therefore be used to create a common comprehension - interpretable by human beings and ma- 
chines - of a specific domain, e.g. the commodity groups of a particular industrial sector and their properties [Din+02, p. 211]. Formal languages to describe ontologies enhance the basic concept of RDF. Among those languages, the most promising one is DAML+OIL (see http://www.daml.org/language/).

What benefit can the Semantic Web deliver to the user community in the field of business process integration? As described in the following, semantic technologies form a building block in the component model that addresses the problem of a machine readable representation of business process expertise and domain knowledge, hence provide a contribution to the model's completeness.

\section{Development of the Integrated Model}

The following chapter deals with the development of a component model for interorganizational business process integration. The development starts with the identification of the model's requirements. Due to its complexity, the model is decomposed into different phases before the parts of the model are introduced in greater detail.

\subsection{Model Requirements}

The requirements for the component model derive both from the market needs as introduced in Section 2 and the shortcomings of existing approaches as outlined in Section 3. These requirements are at a glance:

- Automated integration and execution of business processes: On the one hand, the model must support the automation of the integration process so that this task can be taken over by information systems to an extent that reduces manual efforts significantly. On the other hand, the model must also support the execution of integrated processes.

- High flexibility: In order to support dynamic networks, the model must provide users with mechanisms to integrate their processes 'on the fly', i.e. in an easy, fast manner.

- Inter-organizational applicability: True inter-organizational applicability means that the model must also support highly dynamic business processes that might change during runtime and that are beyond the control of the trading partners.

- Ease of use: In order to be applicable both for large companies and SMEs, the model must be based on widely accepted standards and easy to implement. 
- Neutrality: The model should make use of technologies and standards that are available free of charge and do not depend on the interests of any commercial organizations.

\subsection{Model Decomposition}

Looking at the requirements, it becomes evident that the model to be developed must be of high complexity. In order to cope with this complexity, the model will not be developed as a monolithic block, but will rather be decomposed in separate layers that allow different views on the model. The proposed component model for efficient business process integration consists of three different layers, namely the 'integration phases' layer, the 'levels of implementation' layer and the 'actor roles' layer. These layers are described in greater detail in the following.

\subsubsection{Integration Phases}

The integration phases take into account that business process integration does not stop with the execution of business transactions but should also consider the integration process itself. Therefore, four different phases can be identified as depicted in Figure 3 [cp. open03] $]^{2}$.

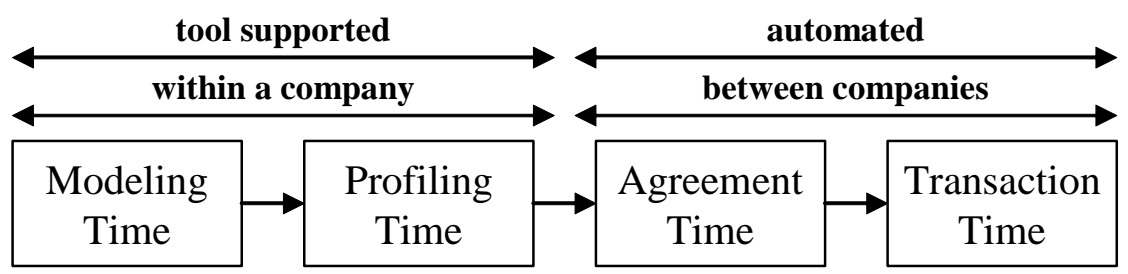

Figure 3: Integration phases

In the modeling phase, an organization models its business processes that are at the interface with external partners, e.g. procurement and sales processes. In doing so, the modeler makes use of existing reference business processes of its domain or industrial sector to make sure that the model can be understood by external partners [cp. Otto02]. The model is then translated - either manually or tool supported - in a machine readable profile according to an appropriate business process framework such as ebXML or RosettaNet (see chapter 3.1). Since the profile is available in a machine readable format, it can be automatically matched with the profile of a trading partner. The result of the agreement process is either a mutually agreed profile that represents the common business process of both trading partners or an 'error log' that contains the differences between the two

2 For a more detailed description refer to [open02b]. 
profiles that are not matchable. In case the matching process does not lead to an agreement, both partners need to adapt their business processes. ${ }^{3}$ If the matching process is successful, an XML based agreement profile is available to both sides that is used to control the business process at runtime [FoOt02, p. 1177].

\subsubsection{Levels of Implementation}

The proposed approach recognizes different levels of implementation in order to close the gap between business process models and implemented processes, hence to facilitate the automation of all four integration phases (see chapter 4.3.1). The different implementation levels are depicted in Figure 4.

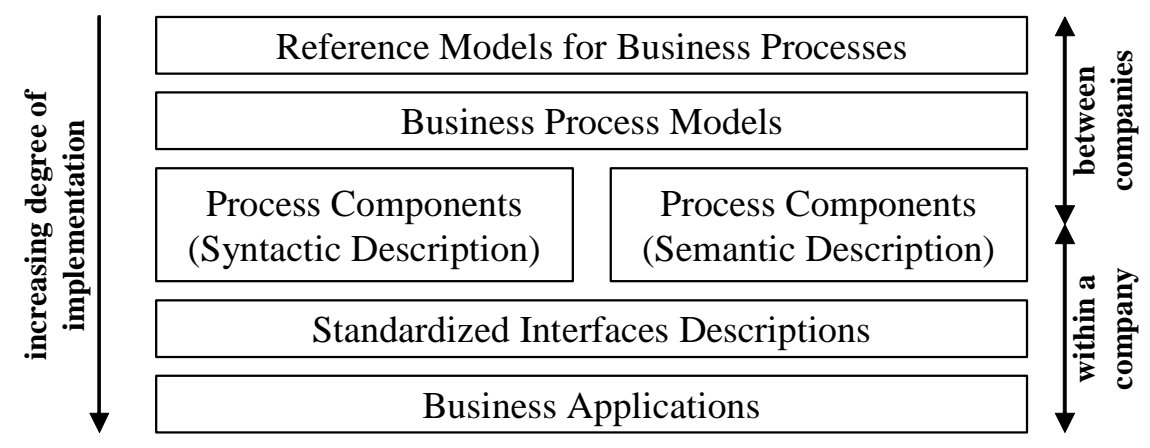

Figure 4: Levels of implementation

The model assumes that all internal business processes relevant also to external partners are supported by business applications such as ERP systems, shop systems or procurement systems. Due to the fact that process integration must consider the existing system landscape and existing API capabilities, the trading partners need to describe the interfaces to their applications using a standardized description language, e.g. WSDL. Standards like WSDL represent certain business functionality as services. These services must correspond to process components.

The process components combine several advantages:

- Due to the 'encapsulated representation' of business functionality, process harmonization can be achieved more easily than with monolithic business processes. End-to-end processes can be assembled in a more flexible way, what leads to a broader acceptance of standardized business processes.

- Process components take advantage of the concept of component based software engineering through the re-use of existing components, the use of proven

3 Of course the trading partners can come to the conclusion that they do not adapt their business processes accordingly. However, in that case automated processes cannot be installed. 
components, short assembly times of large structures of components, and functional diversity [Weis02, p. 147].

- Also according to the component concept in software engineering, process components require exactly defined interfaces [CoHe01, p. 7]. This requirement suits very well the concept of Web Services to describe business functionality as a standardized interface (see chapter 3.3).

The process components have a syntactic and a semantic dimension. XML based specifications form the foundation for a machine readable format of a process component [cp. Otto02]. This would be sufficient for deterministic processes which are known to the trading partners in advance of the integration and which do not include process components that are beyond their control. However, for automated process integration that includes also business processes that are not fully known in advance or that are of dynamic structure during execution, a more sophisticated description of the semantics is required. Therefore, it is necessary to provide also the semantic information of process components in a machine readable format. This goal can be achieved by using semantic technologies - as described in Section 3.4 - or by using grammar concepts to combine syntax and semantics of business processes [Heim02, p. 128].

The business process models that represent the established processes of one or more organizations must consist of the process components defined on the level underneath. In contrast to the latter, end-to-end processes depict business processes from the initial triggering event until the final status that ends the entire process $[$ Pap+00, p. 332]. Taking into account the requirement of closing the gap between the modeling and the implementation view of processes, the concept of end-to-end business processes forms a link between the more business oriented approaches of understanding processes, such as Supply Chain Management, ISO 9000 or Total Quality Management (TQM) concepts, and technology oriented concepts, such as component based application systems. Apart from that, end-toend business processes are better suited than process components to represent also the strategic alignment of entire business functions, e.g. the long-term process improvement in sales and distribution on the condition of an increasing customer orientation [cp. OtBe02].

On an even higher level of abstraction, reference models for business processes comprise the business process knowledge of entire business domains or industrial sectors [Mert01; Sche98]. Therefore, they should work as a starting point to define business processes for common usage that could also be stored in a public process repository. For a broad acceptance within the user community, reference models should be available in standard modeling notations that are part of methodologies as described in Section 3.2. 


\subsubsection{Actor Roles}

In general, the model comprises two different actor roles:

- Trading partners ${ }^{4}$ : Usually companies within supply chains are assigned the role of a trading partner. A trading partner wants to do business efficiently and is therefore willing to integrate its own business processes with those of other business partners. They model and implement their own processes and make use of those parts of the overall approach that are publicly available.

- Registrars: In contrast to trading partners, registrars administer the public parts of the model, i.e. the common reference models, the representation of end-toend business processes and the standardized business process components as described in Section 4.3.2. Registrars operate a registry that is supposed to store the related data and process information.

It is highly relevant to distinguish between these two roles, because in an automated environment trading partners would be users of services that are provided by the service provider, namely the registrar.

\subsection{Model Integration}

Taking into account the objective of the approach to provide a comprehensive model, this section introduces the integration of the different model layers described above. The four integration phases and their relationships to both the levels of implementation and the actor roles form the starting point of the model integration. A solid line in Figure 5 below represents a mandatory relationship, whereas a dashed line stands for an optional relationship that does not necessarily need to be established.

Reference models are mainly used for the modeling time, but they can also support the profiling. End-to-end processes can either be modeled with methodologies, such as ARIS or UMM, or with framework specifications, such as ebXML BPSS. They are used during the modeling time and the profiling time. The process components are used in the last three phases and need to be presented both syntactically - e.g. as ebXML BPSS/CPP, WSFL, or RosettaNet PIPs - and semantically as RDF models. The interface descriptions can be expressed as WSDL and are used for the inter-organizational phases.

The role of the trading partner is certainly involved in all phases, whereas the registrar's role is only mandatory during the profiling time and the agreement time. However, if processes are considered that are beyond the control of the trading partners, the registrar's functionality can also be used to control the process flow.

4 For simplicity reasons no further identification of roles - such as buyer or seller - is made here. 


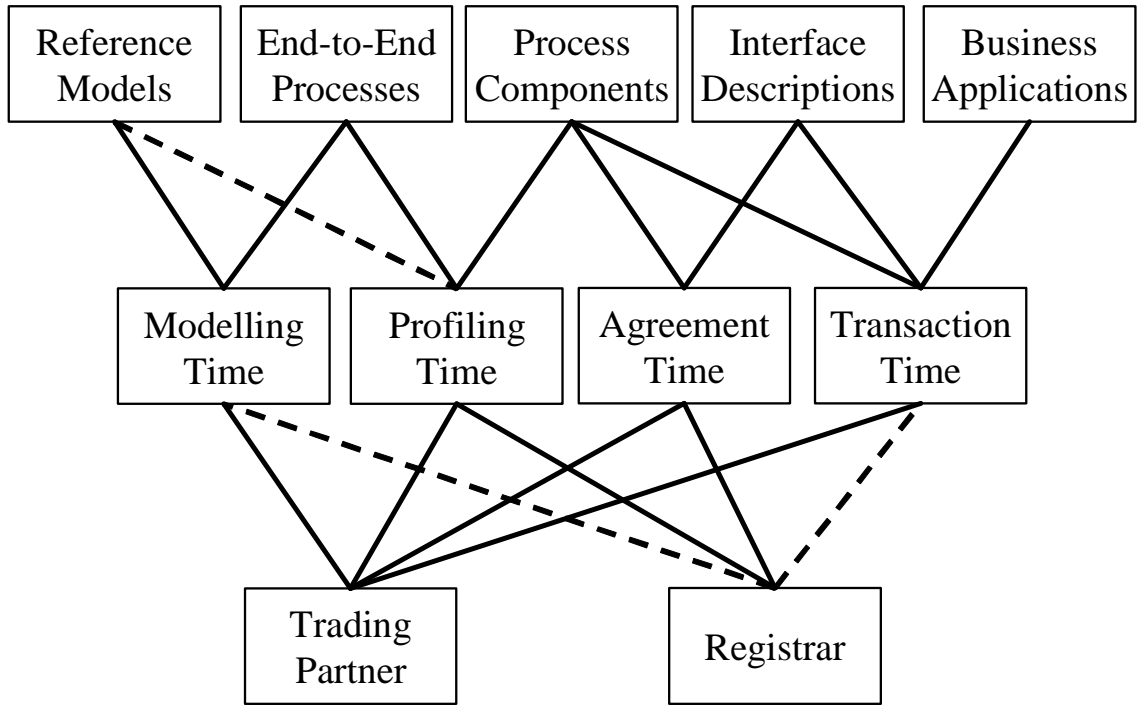

Figure 5: Integration of the model layers

\section{Potential Benefits and Conclusions}

The approach proposed in this paper introduces an integrated model for business process integration and automation, covering all phases of the integration process. It suggests how state-of-the-art technologies, such as business process frameworks, Web Services and the Semantic Web, can be combined in order to establish cost-effective inter-organizational business process environments. The following considerations form the main benefits for companies that want to improve inter-organizational business processes:

- The proposed model facilitates the integration of truly dynamic business processes, thus enhancing the current scope of integration with a new dimension.

- Based on the easy business process integration, the model supports the reduction of the vertical range of manufacture and the consequent concentration on companies' core competencies.

- Highly flexible and adaptable business processes can be realized across company boundaries at low costs.

- In order to tap the full potential of the proposed model, a lot of contributions and developments are yet to be made. These are e.g.: 
- Industrial companies need to optimize their internal processes to be able to be connected to a dynamic network. Companies should develop internal process templates that could form a foundation for sector wide process frameworks. Companies - and also industry associations - should seek to actively influence the standardization process. Also should the processes and the underlying systems be developed in the most open way possible in order to flexibly be able to cope with future requirements (both syntactical and semantic ones).

- Research organizations should take care of the applicability of the different model components and should also provide utilization guidelines to increase the acceptance of the proposed standardized components in the user community. Moreover, research organizations should form a linkage point between industry needs and standardization activities. Also, a lot of research is yet to be done regarding process reference models which are far from providing a level of detail and scope that is sufficient for practical use. Moreover, many process reference models are not available in a machine readable format.

- Regulatory bodies and governmental institutions should provide the general conditions for the efficient establishment of the proposed model components. Regarding the registrars' role e.g., a comprehensive regulatory framework is needed for security and quality aspects and for data privacy and protection aspects. Moreover, regulatory bodies should give recommendations what type of organizations should operate the common registries. Should it be non-profit organizations or vendors that will be equipped with a certain 'mandate'?

At present, the profiling, agreement and transaction phases of the proposed model are being implemented and verified within the context of the EU funded openXchange project.

\section{Acknowledgement}

- This work has been partially funded by the European Commission through the openXchange project (IST 2000-28548). The authors would like to acknowledge the European Commission for its support and to express their appreciation to all the openXchange project partners for their contribution during the development of various ideas and concepts presented in this paper. 


\section{References}

[And+03] Andrews, T. et al.: Business Process Execution Language for Web Services Version 1.1. ftp://www6.software.ibm.com/software/developer/library/ws-bpel11.pdf, 2003, Request on 2003-05-14.

[Ark+02] Arkin, A. et al. : Web Service Choreography Interface 1.0. http://wwws.sun.com/ software/xml/developers/wsci/wsci-spec-10.pdf, 2002, Request on 2003-05-14.

[Bett01] Bettag, U.: Web-Services. In: Informatik-Spektrum (2001) Vol. 24 No. 5, p. 302304.

[BrGu03] Brickley, D.; Guha, R. V.: RDF Vocabulary Description Language 1.0: RDF Schema. http://www.w3.org/TR/rdf-schema/, 2003, Request on 2003-02-14.

[BuFä97] Bullinger, H.-J.; Fähnrich, K.-P.: Betriebliche Informationssysteme: Grundlagen und Werkzeuge der methodischen Softwareentwicklung. Berlin, Heidelberg: Springer, 1997.

[Bul199] Bullinger, H.-J.: Turbulent times require creative thinking: New European concepts in production management. In: International Journal of Production Economics (1999) No. 60-61, p. 9-27.

[BuSc01] Business Process Management Tools. Eine evaluierende Marktstudie über aktuelle Werkzeuge. Stuttgart: IRB, 2001.

[Chi+03] Chinnici, R.; Gudgin, M.; Moreau, J.-J.; Weerawarana, S.: Web Services Description Language (WSDL) Version 1.2. http://www.w3.org/TR/2003/WD-wsdl1220030124, 2003, Request on 2003-02-12.

[CoHe01] Councill, B.; Heinemann, G. T.: Definition of a Software Component and Its Elements. In: Heinemann, G. T.; Councill, B. (Eds.): Component-Based Software Engineering. Boston et al.: Addison-Wesley, 2001, p. 5-19.

[Din+02] Ding, Y.; Fensel, D.; Klein, M.; Omelayenko, B.: The semantic web: yet another hip? In: Data \& Knowledge Engineering (2002) Vol. 41, p. 205-227.

[EsZu02] Esswein, W.; Zumpe, S.: Realisierung des Datenaustauschs im elektronischen Handel. In: Informatik-Spektrum (2002) Vol. 25 No. 8, p. 251-261.

[Fen+01] Fennema, P.; Koolwaaij, J. W.; Lankhorst, M.: Web Services - About to integrate distributed applications? https://doc.telin.nl/dscgi/ds.py/Get/File-18739, 2001, Request on 2003-02-12.

[FoOt02] Folmer, E.; Otto, B.: Smoothening inter-organisational processes with openXchange; a standards-based demonstration and validation. In: Stanford-Smith, B. (Ed.): Challenges and Achievements in E-Business and E-Work. Burke: IOS Press, 2002, p. 1174-1181 (Vol. 2).

[Fran01] Frank, U.: Standardisierungsvorhaben zur Unterstützung des elektronischen Handels: Überblick über anwendungsnahe Ansätze. In: Wirtschaftsinformatik (2001) Vol. 43 No. 3, p. 283-293. 
[Gud+02] Gudgin, M.; Hadley, M.; Mendelsohn, N.; Moreau, J.-J.; Nielsen, H. F.: SOAP Version 1.2 Part 1: Messaging Framework. http://www.w3.org/TR/2002/CR-soap12part1-20021219, 2002, Request on 2003-02-12.

[Heim02] Heimig, I.: Grammatikbasierte Beschreibung von Geschäftsprozessen: Methodik für das strukturierte Verarbeiten von Modellen. Wiesbaden: Deutscher UniversitätsVerlag, 2002.

[Kli+99] Klingemann, J.; Wäsch, J.; Aberer, K.: Adaptive Outsourcing in CrossOrganizational Workflow. CAiSE 1999, p. 417-421.

[Kur+97] Kurbel, K.; Nenoglu, G.; Schwarz, C.: Von der Geschäftsprozessmodellierung zur Workflow-Spezifikation - Zur Kompatibilität von Modellen und Werkzeugen. In: HMD - Praxis der Wirtschaftsinformatik (1997) Vol. 34 No. 198, p. 66-82.

[LaSw99] Lassila, O.; Swick, R. R.: Resource Description Framework (RDF) Model and Syntax Specification. http://www.w3.org/TR/1999/REC-rdf-syntax-19990222, 1999, Request on 2003-02-14.

[Leym01] Leymann, F.: Web Services Flow Language (WSFL 1.0). http://www.ibm.com/ software/solutions/webservices/pdf/WSFL.pdf, 2001, Request on 2003-02-12.

[Mert01] Mertens, Peter: Integrierte Informationsverarbeitung. Bd. 1: Operative Systeme in der Industrie. Wiesbaden: Gabler, 2001.

[Micr02] Microsoft Corporation: BizTalk Framework 2.0: Document and Message Specification. http://www.microsoft.com/biztalk/techinfo/BizTalkFramework20.doc, 2002, Request on 2003-02-12.

[OMG01] Object Management Group, Inc.: OMG Unified Modeling Language Specification. Version 1.4. http://www.omg.org/cgi-bin/doc?formal/01-09-67.pdf, 2001, Request on 2003-02-12.

[open02a] openXchange consortium: State of the Art - Business Process Modelling and EBusiness-Standards. IST-2000-28548 openXchange project deliverable D2.1, 2002.

[open02b] openXchange consortium: Business Design for Prototype Component. IST2000-28548 openXchange project deliverable D.3.1, 2002.

[open03] openXchange consortium: openXchange Overview. http://www.openxchange.org, 2003, Request on 2003-02-14.

[OtBe02] Otto, B.; Beckmann, H.: A customer-oriented approach to reduce the complexity of business process integration. In: Pawar, K. S. (Ed.): Ubiquitous engineering in the collaborative economy : Proceedings of the 8th International Conference on Concurrent Enterprising, Rome, Italy, 17 - 19th June 2002. Nottingham : Univ. of Nottingham, 2002.

[Otto02] Otto, B.: Referenzmodell zur Automatisierung zwischenbetrieblicher Beschaffungsprozesse. Jost Jetter: Heimsheim, 2002. 
[Pap+00] Papazoglou, M. P.; Ribbers, P.; Tsalgatidou, A.: Integrated value chains and their implications from a business and technology standpoint. In: Decision Support Systems (2000) Vol. 29, p. 323-342.

[Petr62] Petri, C.A.: Kommunikation mit Automaten. Bonn: Schriften des Instituts für Instrumentelle Mathematik Nr. 2, 1962.

[Rose00] Rosemann, M.: Vorbereitung der Prozessmodellierung. In: Becker, J. (Ed.): Prozessmanagement - ein Leitfaden zur prozessorientierten Organisationsgestaltung. Berlin, Heidelberg: Springer, 2000, p. 45-90.

[Rose02] RosettaNet: Welcome to RosettaNet - Standards. http://www.rosettanet.org/ standards, 2002, Request on 2003-02-12.

[ScFi02] Schoder, D.; Fischbach, K.: Peer-to-Peer - Anwendungsbereiche und Herausforderungen. In: Schober, D.; Fischbach, H.; Teichmann, R. (Eds): Peer-to-Peer: ökonomische, technologische und juristische Perspektiven. Sprinbger: Berlin et al., 2002, p. 3-21.

[Sche98] Scheer, A.-W.: ARIS - Modellierungsmethoden, Metamodelle, Anwendungen. Berlin et al.: Springer, 1998.

[ScTü99] Schwarz, K.; Türker, C.: Wechselwirkungen und Abhängigkeiten in transaktionalen Workflows. In: Scheer, A.-W.; Nüttgens, M. (Eds.): Electronic Business Engineering. Heidelberg: Physica, 1999, p. 521-542.

[Somm02] Sommergut, W.: Wird ebXML globaler B-to-B-Standard? Computerwoche Online. http://www.computerwoche.de/index.cfm?pageid=255\&artid=27133\&type= detail\&category=160, 2002, Request on 2003-02-12.

[Stef02] Stefansson, G.: Business-to-business data sharing: A source for integration of supply chains. In: International Journal of Production Economics (2002) No. 75, p. 135146.

[UN/C01] UN/CEFACT - Business Process Analysis Working Group (BPWAG): UN/CEFACT modelling methodology. http://www.unece.org/cefact/docum/download/ 01bp_n090.zip, 2001, Request on 2003-02-12.

[VDA02] Verband der Automobilindustrie e.V.: Auto Jahresbericht 2002. Druckerei Henrich GmbH: Frankfurt am Main, 2002.

[W3C03] World Wide Web Consortium: Web Services Activity. http://www.w3.org/ 2002/ws/, 2003, Request on 2003-02-12.

[WeCu02] Weerawarana, S.; Curbera, F.: Business Processes: Understanding BPEL4WS, Part 1. ftp://www6.software.ibm.com/software/developer/library/ws-bpelcol1.pdf, 2002, Request on 2003-02-12.

[Weis02] Weisbecker, A.: Software-Management für komponentenbasierte SoftwareEntwicklung. Heimsheim: Jost Jetter, 2002. 
[WoWe02] Wojciechowski, R.; Weinhardt, C.: Web Services and Peer-to-Peer-Netzwerke. In: Schoder, D.; Fischbach, K.; Teichmann, R. (Hrsg.): Peer-to-Peer: ökonomische, technologische und juristische Perspektiven. Springer: Berlin et al., 2002, p. 99-117. 